\title{
Decision tree-based classifiers for lung cancer diagnosis and subtyping using TCGA miRNA expression data
}

\author{
MASIH SHERAFATIAN $^{1}$ and FATEME ARJMAND ${ }^{2}$ \\ ${ }^{1}$ Department of Molecular Genetics, Faculty of Biological Sciences, Tarbiat Modares University, Tehran 14115-111; \\ ${ }^{2}$ Department of Genetics and Molecular Medicine, Zanjan University of Medical Sciences, Zanjan 45139-56184, Iran
}

Received October 11, 2018; Accepted March 19, 2019

DOI: 10.3892/ol.2019.10462

\begin{abstract}
Lung cancer has the world's highest cancerassociated mortality rate, making biomarker discovery for this cancer a pressing issue. Machine learning approaches to identify molecular biomarkers are not as prevalent as screening of potential biomarkers by differential expression analysis. However, several differentially expressed miRNAs involved in cancer have been identified using this approach. The availability of The Cancer Genome Atlas (TCGA) allows the use of machine-learning methods for the molecular profiling of tumors. The present study employed empirical negative control microRNAs (miRs) in lung cancer to normalize lung adenocarcinoma (LUAD) and lung squamous cell carcinoma (LUSC) datasets from TCGA to model decision trees in order to classify lung cancer status and subtype. The two primary classification models consisted of four miRNAs for lung cancer diagnosis and subtyping. hsa-miR-183 and hsa-miR-135b were used to distinguish lung tumors from normal samples taken from tissues adjacent to the tumor site, and hsa-miR-944 and hsa-miR-205 to further classify the tumors into LUAD and LUSC major subtypes. Specific cancer status classification models were also presented for each subtype.
\end{abstract}

\section{Introduction}

Lung cancer was reported to have the world's leading cancer-associated mortality in 2008 (1). It is classified into two main histological subtypes: Non-small cell lung cancer (NSCLC), which accounts for $\sim 85 \%$ of cases, and small cell lung cancer (SCLC) (2). NSCLC is further subdivided into lung squamous cell carcinoma (LUSC) and lung adenocarcinomas (LUAD), which generally affect the epithelial cells

Correspondence to: Mr. Masih Sherafatian, Department of Molecular Genetics, Faculty of Biological Sciences, Tarbiat Modares University, 7 Al Ahmad Street, Tehran 14115-111, Iran

E-mail: sherafatian@modares.ac.ir

Key words: lung cancer, miRNA biomarker, diagnosis, subtyping, The Cancer Genome Atlas lining the larger airways and the peripheral smaller airways, respectively (3). The majority of patients with lung cancer are diagnosed at advanced stages and consequently the five-year survival rate is $16.8 \%$ (4). The identification of new diagnostic strategies is therefore required to reduce lung cancer-associated mortality (5).

MicroRNAs (miRNAs/miRs) are small, stable, single-stranded non-coding RNAs which are present in tissues and body fluids (6). These molecules have been revealed to serve roles in the mechanisms underlying cancer initiation and progression (7-9). Previous studies have demonstrated the potential use of miRNAs in the non-invasive detection of LOAD (2) as well as in the classification of diverse histological subtypes and identification of the source tissue in cases of poorly differentiated tumors (10).

Machine learning may be used as an alternative approach to statistical methods including differential expression analysis (11). There are two main types of machine learning algorithms: Supervised, where the algorithm is given some prior knowledge, and unsupervised, where it is not given any prior information. The most common applications of unsupervised and supervised learning are clustering and discriminant analysis, respectively. A decision tree is a type of supervised machine learning algorithm used for discriminant analysis, which is simple to understand and interpret. It allows the extraction of knowledge from data by generating understandable knowledge structures in the form of hierarchical trees or sets of rules and presenting them in a graphically intuitive way. Attributes which are important for prediction or classification are subsequently selected with a low computational cost. A decision tree is a greedy algorithm constructed by a step-by-step process called recursive partitioning which is also known as hierarchical classification. The dataset is divided into training and testing data and the training data are subsequently used to create the decision tree model and test its performance (12). Several studies have used decision trees to solve biological problems, including predicting the expression status using chromatin modifications in the Encyclopedia of DNA Elements pilot project (13) and identifying cancer tissue origin using microRNAs $(14,15)$. Decision trees have also been used to identify biomarkers in cancer, including defining a set of prognostic biomarkers for lung cancer using nuclear receptor expression (16). Although less widely used compared with differential analysis methods, the decision tree method 
is applicable in cancer classification using gene expression data (17).

The present study aimed to identify lung cancer diagnostic and subtyping biomarkers by applying the decision tree method to the largest publicly available repository of miRNA expression in lung cancer collected by The Cancer Genome Atlas (https://portal.gdc.cancer.gov/).

\section{Materials and methods}

All calculations and plotting associated with data retrieval, preprocessing, filtering, differential expression, normalization, handling class imbalance, and applying and evaluating machine learning algorithms were performed in $\mathrm{R}$ (version 3.5.0; The R Foundation for Statistical Computing; http://www.r-project. org/foundation) using bioconductor packages (18). The biomarker discovery pipeline used in the current study is the same as previously described (19).

Data retrieval and exploratory data analysis. Level three miRNA sequencing data as well as the clinical dataset were obtained from 1,068 samples from two lung cancer projects (LUAD and LUSC) in TCGA (https://portal.gdc.cancer.gov/). The sequencing data were retrieved and prepared using the TCGAbiolinks package (20). The LUAD project included 499 solid tumor and 46 normal control samples from tissues adjacent to the tumor site, and the LUSC project included 478 solid tumors and 45 normal control samples $(21,22)$.

Filtering features, samples and partitioning datasets. Non-specific filtering was performed by removing miRNAs with expressions of $<100$ reads over at least 10 samples to exclude uninteresting features without regard to the phenotype data and to reduce the number of features that were included in further analysis (19). The genefilter package (23) reduced 1,208 features to 310 features in LUAD and 301 in LUSC. Samples that did not have clinical information in the database were also excluded.

Data were partitioned into training (70\%) and testing (30\%) datasets using the caret package (24) and all subsequent exploratory data analysis and model training were performed only on training datasets. Exploratory data analysis and principal component analysis (PCA) were performed using the EDAseq package (25). PCA plots were drawn in logarithmic scales (Fig. 1).

Normalization and differential expression. The TCGA miRNA expression data were generated through a large collaborative project involving a number of sequencing centers and the data therefore included different batches (19). In order to account for this, the TCGA gene expression data set was normalized using the remove unwanted variation (RUV) normalization method. A set of miRNAs whose expression did not vary across samples, referred to as 'negative controls', were used for the normalization procedure. In order to obtain in silico empirical negative control miRNAs, the $\mathrm{P}$ - and $\mathrm{P}$-adjusted values for all miRNAs were calculated between lung cancer status (normal and tumor) or between its two subtypes (LUAD and LUSC). miRNAs with $\mathrm{P}>0.5$ were selected for further study. These sets of empirical controls were used to remove the factors of unwanted variation and to normalize the data sets for classification. Unwanted variation of raw miRNA sequencing data was removed using the RUVseq package (26), with miRNAs obtained from differential analysis as the internal controls. Least significantly differentially expressed miRNAs obtained using the DESeq 2 package were used as internal negative controls (27). A total of 13 miRNAs in LUSC, 32 miRNAs in LUAD, and 17 and 15 miRNAs in LUAD and LUSC, respectively, were used as internal controls for classification of cancer status and subtypes. Factors of unwanted variation, $\mathrm{k}=1$, were considered in all calculations.

Combating imbalance, tree model training, evaluation and plotting. Class imbalance in training and testing datasets were addressed separately using the Synthetic Minority Oversampling Technique (SMOTE) (28) in the DMwR package (29). Supervised classification was performed using Recursive Partitioning and Regression Trees (RPART), and was implemented using the RPART package (version 4.1-13). Decision trees from the RPART model were plotted using the rattle package version 5.1.0 (30,31). This was followed by adjusted pruning which improves decision tree accuracy by avoiding over-fitting to the training set and reducing its size. The complexity parameter by which the RPART objects trimmed was 0.001 .

ROC curve analyses. ROC curve analyses were performed in $\mathrm{R}$ version 3.5.0 (The $\mathrm{R}$ Foundation for Statistical Computing, http://www.r-project.org/foundation) using procedures from the pROC (32) package.

\section{Results}

Least significantly differentially expressed miRNAs are used for normalization and removal of technical variability. Correction of unwanted variation in data was required in order to fulfill the assumption that the biological variation of interest was the main source of variation in the current study. Since miRNA sequencing expression data obtained from TCGA involved multiple laboratories, unwanted variation dominated the data. The RUV method using in silico empirical controls obtained by differential expression analysis was used for normalization of the data in the present study. The RUV allows the removal of laboratory-specific effects to allow the combined analysis of miRNA sequencing expression changes (26).

Four sets of empirical negative controls based on differential expression analysis were used in the current study. First-pass differential expression analysis was performed in LUAD, LUSC and in combined subtypes separately. Cancer status and cancer subtype were used to obtain least significantly differentially expressed miRNAs. miRNAs with $\mathrm{P}>0.5$ were selected as negative controls. Two sets of empirical negative controls were obtained in combined subtypes (LUAD and LUSC) under two sets of conditions: Cancer status and cancer subtype (Table I).

Exploratory data analysis ensures proper clustering of samples following RUV normalization. PCA was performed following data normalization. PCA plots are an established 


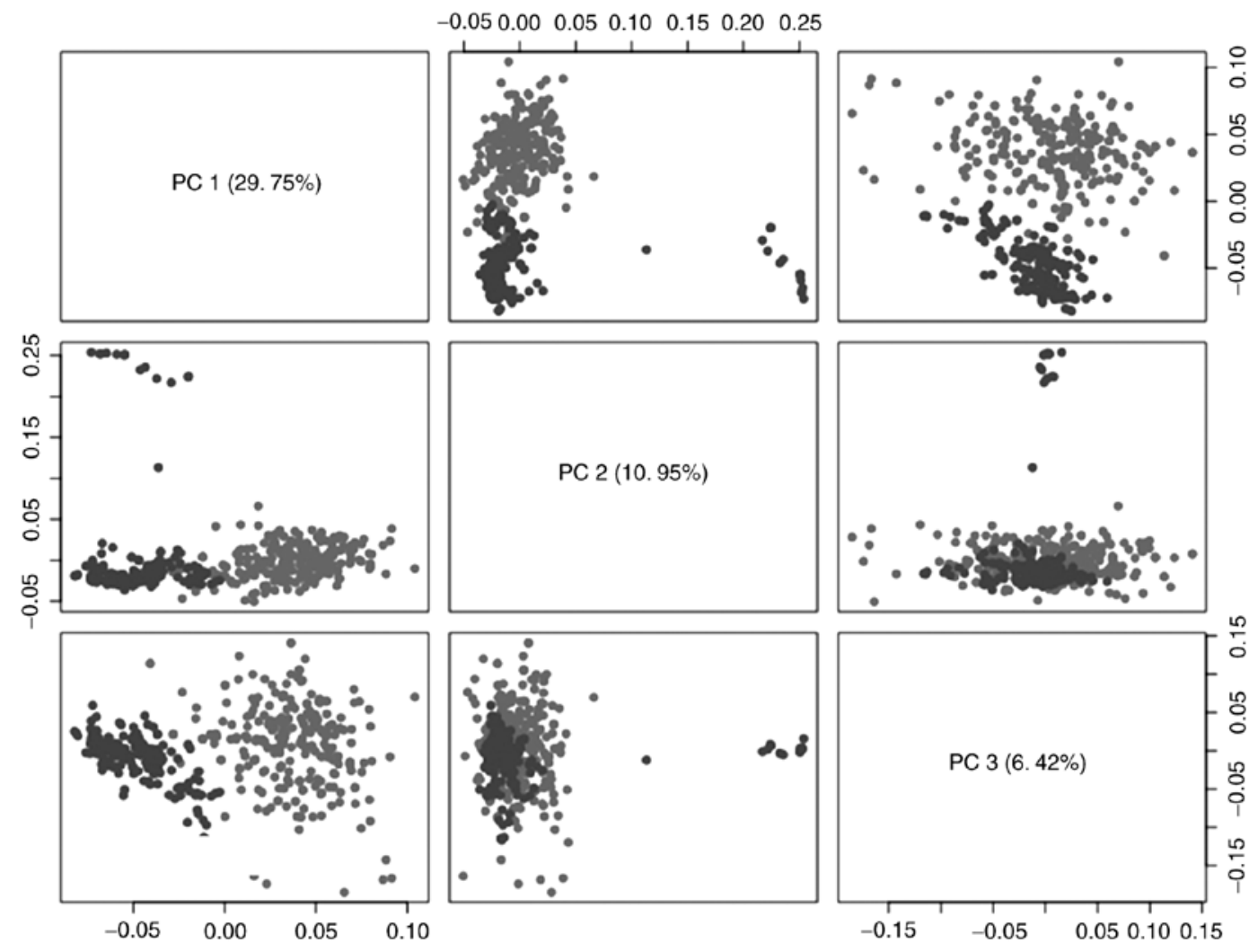

Figure 1. PCA matrix plot of miRNA expression in samples obtained from patients with lung cancer in TCGA database. Three main PCs, including PC1, PC2 and PC3, of miRNA expression in LUAD and LUSC from TCGA database were plotted and colored according to their cancer status (light and dark gray represent cancer and normal, respectively). Each PC is a linear combination of normalized miRNA sequencing counts. In this plot, the diagonal cells specify the axes (PC1, PC2 and PC3) of the remaining cells of the plot. The samples were projected into a lower dimensional space and clustered by their cancer status. LUAD, lung adenocarcinoma; LUSC, lung squamous cell carcinoma; TCGA, The Cancer Genome Atlas; PCA, principal component analysis; PC, principal component.

method of visualizing the sources of variation in genome-wide studies. PCA plots identify the principal components of data by reducing its dimensions. The first principal component (PC1) explains the highest amount of variance across all samples (26). The clustering of samples by the biological factor of interest such as cancer status in the space of main principal components indicated that the data in the present study had clear separation values based on the cancer status prior to classification. Plotting the miRNA expression in first principal component and coloring by the biological factor, cancer status indicated that this was the main driver of clustering in PC1 which avoids false positives and false negatives in the current results (Fig. 1). The first principle component (PC) with highest variation (29.75\%) separated normal lung tissues from cancerous lung tissues. The second and third PCs account for 10.95 and $6.42 \%$ of variation respectively.

Modeling lung cancer miRNA sequencing data with decision tree algorithms identifies complementary diagnostic and subtyping miRNA markers in lung cancer. Due to the predominance of tumor samples over non-tumor samples in TCGA data, the data in the current study were imbalanced. Imbalanced data may affect model training and its subsequent performance (24). Therefore, TCGA data are not suitable for machine learning algorithms to classify cancer status. The imbalance of TCGA data was addressed by SMOTE before training the models to classify cancer status. Using SMOTE, the minority class (normal cases in tumor-normal classification) is over-sampled by creating synthetic samples. Training and testing datasets for classification as tumor or normal were subjected to this approach separately. The PCA plot method was used to ensure the retention of separation of samples with different cancer status.

To classify lung cancer status and its subtypes, two simple models were obtained by applying the RPART algorithm to the balanced miRNA sequencing training datasets. Each model consisted of two essential miRNAs as the primary aim of the current study was to identify the minimal number of biomarkers that can be used to classify lung cancer status and its subtypes.

The main resulting cancer classifier structures were two trained two-step decision trees. The first classification tree distinguished tumor from non-tumor samples in both subtypes of lung cancer (LUAD and LUSC) from TCGA database. Two decision nodes of this classifier are hsa-miR-183 and hsa-miR-135b (Fig. 2). Performances of all classification trees were measured using testing datasets (30\% of total data). Discriminative power of this classifier was then measured by the area under the curve (91.2\%).

More specific classification trees were also trained in each subtype. The classification tree in the LUAD subtype used simple rules to classify tumor status. hsa-miR-183 and hsa-let-7a were two nodes of this decision tree. LUAD samples were classified as normal if hsa-miR-183 and hsa-let-7a expression levels were 
Table I. Least significantly differentially expressed miRNAs in lung cancer considering status of cancer or its subtypes.

A, Cancer status control miRs

\begin{tabular}{lcc}
\hline miR & Lfc & Adjusted P-value \\
\hline hsa-mir-6718 & -0.155 & 0.546 \\
hsa-mir-23a & -0.047 & 0.573 \\
hsa-mir-330 & 0.067 & 0.573 \\
hsa-mir-6720 & -0.136 & 0.573 \\
hsa-mir-181b-2 & -0.066 & 0.606 \\
hsa-mir-5683 & -0.117 & 0.709 \\
hsa-mir-363 & 0.076 & 0.711 \\
hsa-mir-3074 & -0.056 & 0.732 \\
hsa-mir-132 & -0.034 & 0.756 \\
hsa-mir-30e & 0.024 & 0.764 \\
hsa-mir-25 & 0.018 & 0.854 \\
hsa-mir-181b-1 & -0.017 & 0.893 \\
hsa-mir-27b & -0.010 & 0.915 \\
hsa-mir-151a & 0.010 & 0.927 \\
hsa-mir-92b & 0.011 & 0.943 \\
hsa-mir-3130-1 & 0.010 & 0.954 \\
hsa-mir-181d & 0.003 & 0.981 \\
\hline
\end{tabular}

B, Subtype-specific control miRs

\begin{tabular}{lcc}
\hline miR & Lfc & Adjusted P-value \\
\hline hsa-mir-145 & 0.040 & 0.538 \\
hsa-mir-200a & -0.044 & 0.577 \\
hsa-mir-1468 & -0.049 & 0.578 \\
hsa-mir-374a & 0.022 & 0.696 \\
hsa-mir-187 & 0.058 & 0.709 \\
hsa-mir-34b & 0.050 & 0.755 \\
hsa-mir-889 & -0.035 & 0.771 \\
hsa-let-7c & -0.024 & 0.773 \\
hsa-mir-369 & 0.023 & 0.846 \\
hsa-mir-3677 & 0.016 & 0.869 \\
hsa-mir-15a & -0.007 & 0.893 \\
hsa-mir-522 & 0.034 & 0.904 \\
hsa-mir-3613 & -0.006 & 0.932 \\
hsa-mir-2355 & -0.005 & 0.939 \\
hsa-mir-485 & 0.002 & 0.988 \\
\hline
\end{tabular}

miR/miRNA, microRNA; adj, adjusted; lfc, logarithm of fold change; LUSC, lung squamous cell carcinoma; LUAD, lung adenocarcinoma.

$<12 \times 10^{3}$ and $\geq 68 \times 10^{3}$, respectively. Area under the curve for this classifier was $95.1 \%$. The classification tree in the LUSC subtype used hsa-miR-30a and hsa-miR-1269a to classify tumors from non-tumors. Samples were classified as normal if the expression levels of hsa-miR-30a and hsa-miR-1269a were $>134 \times 10^{3}$ and $<48$, respectively. The discriminative power of this classification tree was measured after testing this model on the testing dataset (data not shown). The area under the curve was $95.2 \%$. A further tree distinguished lung cancer subtypes (LUAD from LUSC) using two miRNAs, hsa-miR-944 and hsa-miR-205. A sample was classified as LUSC if the expression levels of hsa-miR-944 and hsa-miR-205 were $<80$ and $<3,376$, respectively. Area under the curve for this classifier was $91.6 \%$ (Fig. 3). The four miRNAs used to classify lung cancer status and its subtypes in the current study were: hsa-miR-183, hsa-miR-135b, hsa-miR-944 and hsa-miR-205.

\section{Discussion}

The current study used 5 miRNAs in the diagnosis of lung cancer for three separate classifications: miR-183 and let-7a in the LUAD subtype, miR-30a and miR-1296a in the LUSC subtype and miR-183 and miR-135b in both subtypes. Let-7a, one of the two biomarkers used in the LUAD model of cancer diagnosis in the present study, is a member of the let-7 family and one of the first miRNAs implicated in of lung cancer. Takamizawa et al (7) reported that let-7 expression was lower in lung cancer compared with healthy control tissue and that lower expression of let-7 was associated with poor prognosis. Furthermore, the overexpression of let-7 in the A549 lung adenocarcinoma cell line inhibited cell growth, and was revealed to act as a tumor suppressor by decreasing cell proliferation and regulating oncogenes including tumor protein $\mathrm{p} 53$, RAS type GTPase family (RAS) and MYC proto-oncogene bHLH transcription factor $(5,33)$. High expression of let-7 and downregulation of its target oncogenes (high mobility group AT-hook 2 and RAS) in well-differentiated lung tumors suggested that let-7 may be a biomarker for poorly differentiated tumors (33). Landi et al (3) analyzed 440 human miRNAs and identified a signature consisting of five miRNAs, including hsa-let-7b, that differentiated LUAD from LUSC and had prognostic value.

The miR-183 family members, including miR-182, miR-183 and miR-96, exhibit oncogenic and tumor suppressor functions in different types of cancer. miR-183 inhibited lung tumor invasion and metastasis by targeting ezrin. Overexpression of miR-183 was reported as a risk factor for lung cancer by Feng et al (34). Sun et al (35) reported that the overexpression of miR-126 in non-small cell lung cancer cells resulted in decreased cell proliferation in vitro and tumor growth in vivo. Zhong et al (36) revealed dose-dependent inhibition of lung cancer cell growth by miR-107, miR-126 and let-7a in vivo, suggesting that the overexpression of these miRNAs may suppress cancer.

Leidinger et al (4) analyzed 74 individual whole blood samples and revealed that miR-20b-5p, miR-20a-5p, miR-17-5p and miR-106a-5p accurately differentiated patients with NSCLC from unaffected controls with a specificity and sensitivity of 98 and $91 \%$, respectively. Su et al (37) analyzed sputum samples from 103 patients with NSCLC and 528 cancer-free smokers. The authors identified a panel of three sputum miRNA biomarkers (miRs-21, -31 and -210) with $83 \%$ sensitivity and $88 \%$ specificity for the early detection of lung cancer. Võsa et al (38) performed a meta-analysis of 20 published miRNA expression studies in lung cancer and identified seven upregulated (miR-21, miR-31, miR-182, miR-183, miR-200b, miR-210 and miR-205) and eight downregulated (miR-30a, miR-30d, miR-126-3p, miR-126-5p, 

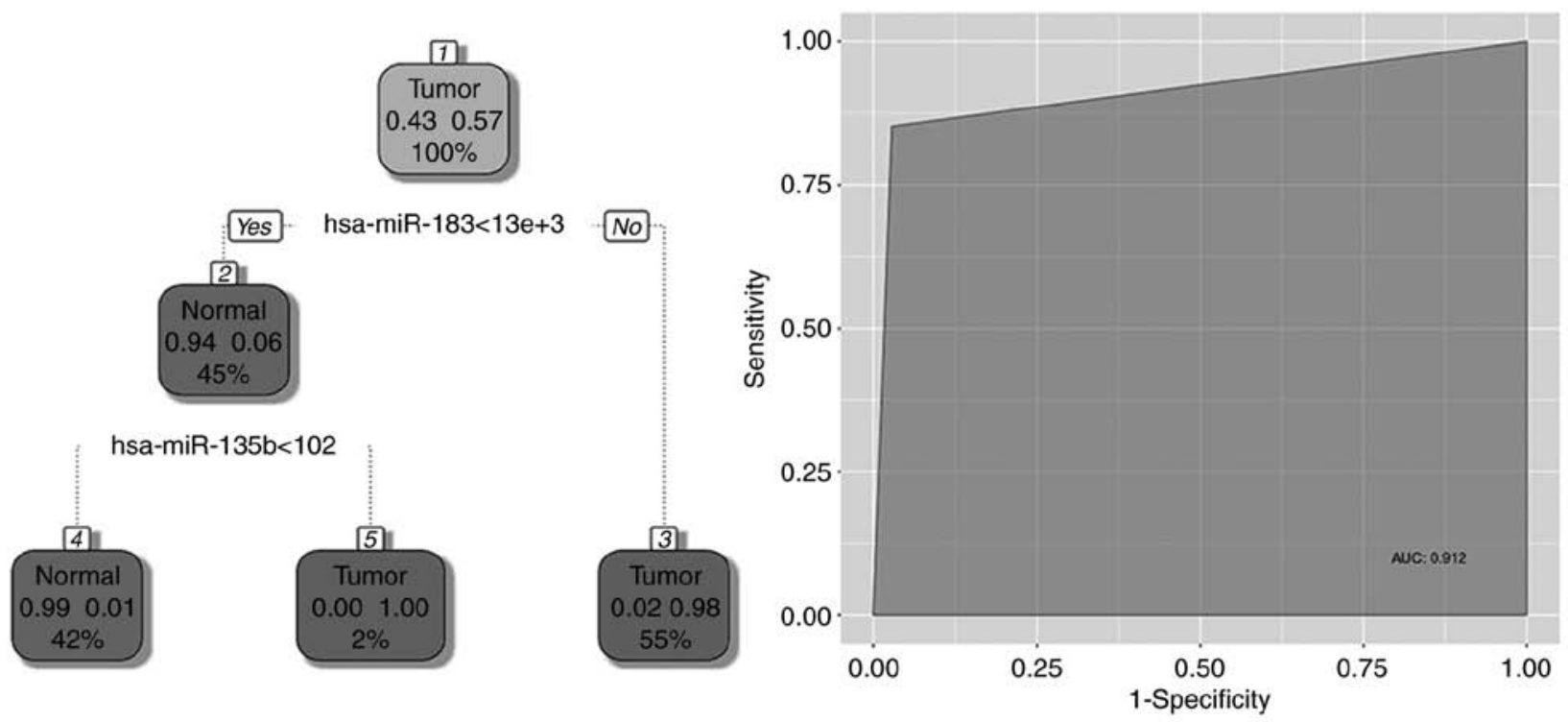

Figure 2. RPART classification tree and its performance in classification of lung cancer tissues from normal tissues based on miR sequencing data from TCGA following normalization. LUAD and LUSC data from TCGA were merged and a decision tree trained with RPART algorithm on 70\% of balanced and normalized miRNA expression was generated. The decision tree with two miRNA nodes (hsa-miR-183 and hsa-miR-135b) is presented on the left. Normalized counts of these miRNAs were used as decision rules. Performance of this decision tree was obtained from testing the tree model on balanced and normalized testing dataset ( $30 \%$ of data) and presented on the right as a receiver operating characteristic curve (AUC=0.912). miR/miRNA, micro RNA; LUAD, lung adenocarcinoma; LUSC, lung squamous cell carcinoma; AUC, area under the curve; RPART, Recursive Partitioning and Regression Trees; TCGA, The Cancer Genome Atlas.
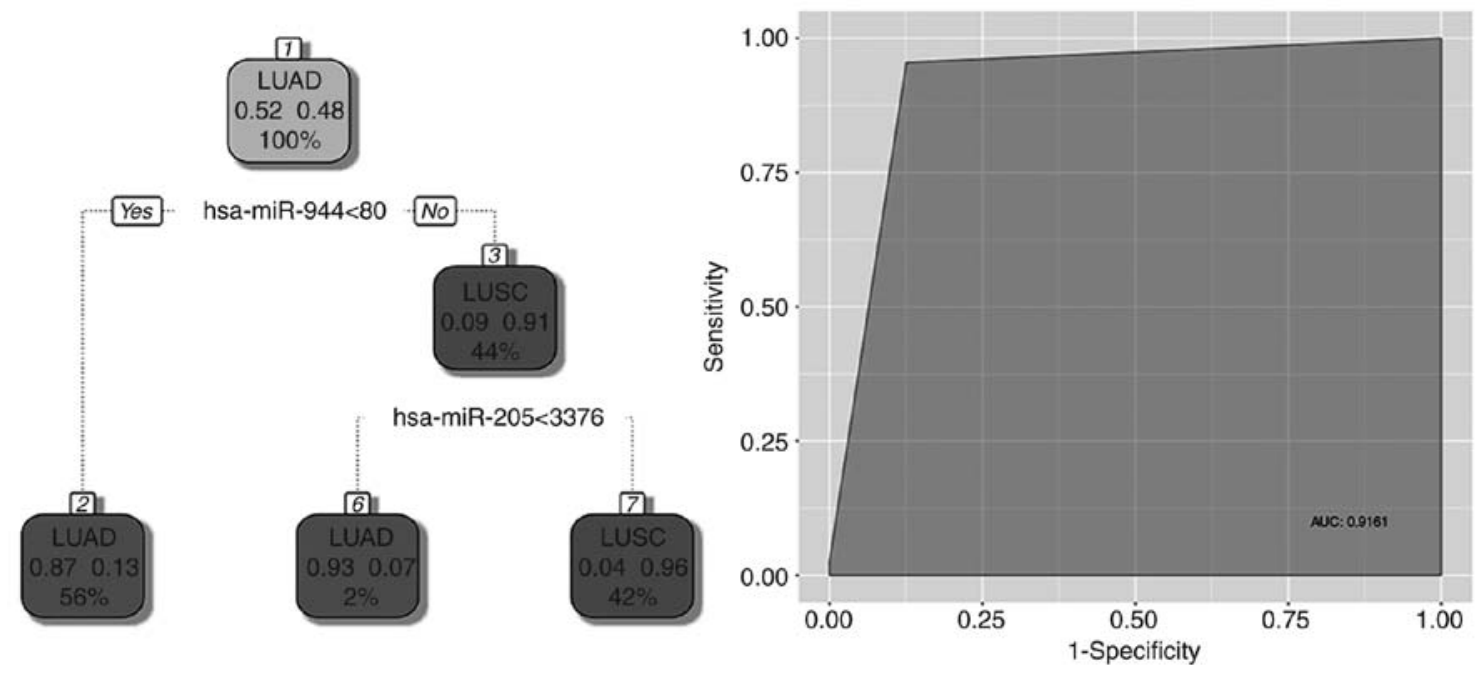

Figure 3. Decision tree and its performance in classification of lung cancer subtypes based on miR sequencing data from The Cancer Genome Atlas following normalization. LUAD and LUSC were classified by decision tree trained with RPART algorithm on 70\% of balanced and normalized miRNA expression. The decision tree with two miRNA nodes (hsa-miR-944 and hsa-miR-205) is presented on the left. Normalized counts of these miRNAs were used as decision rules. Performance of this decision tree was obtained from testing the tree model on balanced and normalized testing dataset (30\% of data) and presented on the right as a receiver operating characteristic curve ( $\mathrm{AUC}=0.916$ ). miR/miRNA, micro RNA; LUAD, lung adenocarcinoma; LUSC, lung squamous cell carcinoma; AUC, area under the curve.

miR-143, miR-145, miR-486-5p and miR-451a) miRNAs as a statistically significant meta-signature of lung cancer.

miR-205 and miR-944 were used as subtyping biomarkers of lung cancer in the current study. miR-205 and miR-21 were previously reported to accurately distinguish LUAD from LUSC subtypes (39). However, miR-205 was subsequently revealed to be useful as an adjunctive diagnostic criterion in selected cases but should not be used as a substitute of accurate morphological and immunophenotypical characterization of lung tumors (10). Lebanony et al (40) reported decreased expression of miR-205 in LUAD compared with LUSC subtypes and suggested that the expression level of miR-205 may be used to predict and diagnose LUSC. Lu et al (41) revealed that the expression of miR-205 in NSCLC may be used to distinguish patients with LUAD from those with LUSC.

Zhang et al (42) assessed the application of miRNA for lung cancer screening and demonstrated that the expression profile 
of sputum miR-21, miR-486, miR-37 and miR-200b yielded $81 \%$ sensitivity and $92 \%$ specificity in distinguishing patients with NSCLC from healthy individuals. Hamamoto et al (43) revealed that the expression profile of miR-205, miR-196b and miR-375 yielded $85 \%$ sensitivity and $83 \%$ specificity in the distinction between patients with LUSC and LUAD.

The miR-200 family and miR-205 have been implicated in the epithelial-mesenchymal transition in a number of breast cancer cell lines by targeting zinc finger E-box-binding protein transcription factors to alter the gene expression of vimentin and E-cadherin (44). Duan et al (45) demonstrated that miR-205 was significantly higher in NSCLC patients. miR-205 had a positive correlation with protein kinase $\mathrm{B}$ gene expression in NSCLC cancer tissues. Increased expression levels of protein kinase B enhanced the invasion abilities of cancer cells.

\section{Acknowledgements}

The authors acknowledge Professor Seyed Javad Mowla (Tarbiat Modares University, Tehran, Iran) for his encouragement and Dr Javad Zahiri (Tarbiat Modares University) for his insights into the manuscript.

\section{Funding}

No funding was received.

\section{Availability of data and materials}

The datasets analyzed during the present study are available in the TCGA repository, (https://portal.gdc.cancer.gov/).

\section{Authors' contributions}

FA was responsible for the literature review and writing the discussion and introduction of the paper. MS was responsible for the bioinformatics analysis, material and methods and results sections of the manuscript.

\section{Ethics approval and consent to participate}

Not applicable.

\section{Patient consent for publication}

Not applicable

\section{Competing interests}

The authors declare that they have no competing interests.

\section{References}

1. Ridge CA, McErlean AM and Ginsberg MS: Epidemiology of lung cancer. Semin Intervent Radiol 30: 93-98, 2013.

2. MacDonagh L, Gray SG, Finn SP, Cuffe S, O'Byrne KJ and Barr MP: The emerging role of microRNAs in resistance to lung cancer treatments. Cancer Trea Rev 41: 160-169, 2015.

3. Landi MT, Zhao Y, Rotunno M, Koshiol J, Liu H, Bergen AW, Rubagotti M, Goldstein AM, Linnoila I, Marincola FM, et al MicroRNA expression differentiates histology and predicts survival of lung cancer. Clin Cancer Res 16: 430-441, 2010.
4. Leidinger P, Brefort T, Backes C, Krapp M, Galata V, Beier M, Kohlhaas J, Huwer H, Meese E and Keller A: High-throughput qRT-PCR validation of blood microRNAs in non-small cell lung cancer. Oncotarget 26: 4611-4616, 2016.

5. Inamura K and Ishikawa Y: MicroRNA in lung cancer: Novel biomarkers and potential tools for treatment. J Clin Med 5: E36, 2016.

6. Anglicheau D, Muthukumar T and Suthanthiran M: MicroRNAs: Small RNAs with big effects. Transplantation 90: 105-112, 2010.

7. Takamizawa J, Konishi H, Yanagisawa K, Tomida S, Osada H, Endoh H, Harano T, Yatabe Y, Nagino M, Nimura Y, et al: Reduced expression of the let-7 microRNAs in human lung cancers in association with shortened postoperative survival. Cancer Res 64: 3753-3756, 2004.

8. Iorio MV and Croce CM: MicroRNA dysregulation in cancer: Diagnostics, monitoring and therapeutics. A comprehensive review. EMBO Mol Med 4: 143-159, 2012.

9. Yanaihara N, Caplen N, Bowman E, Seike M, Kumamoto K, Yi M, Stephens RM, Okamoto A, Yokota J, Tanaka T, et al: Unique microRNA molecular profiles in lung cancer diagnosis and prognosis. Cancer Cell 9: 189-198, 2006.

10. Del Vescovo V, Cantaloni C, Cucino A, Girlando S, Silvestri M, Bragantini E, Fasanella S, Cuorvo LV, Palma PD, Rossi G, et al: miR-205 Expression levels in nonsmall cell lung cancerdo not always distinguish adenocarcinomas from squamous cell carcinomas. Am J Surg Pathol 35: 268-275, 2011.

11. Geurts P, Irrthum A and Wehenkel L: Supervised learning with decision tree-based methods in computational and systems biology. Mol Biosyst 5: 1593-1605, 2009.

12. Safavian SR and Landgrebe DA: A survey of decision tree classifier methodology. IEEE Trans Systems Man Cybernetics 3: 660-674, 1991.

13. ENCODEProjectConsortium,BirneyE,Stamatoyannopoulos JA, Dutta A, Guigó R, Gingeras TR, Margulies EH, Weng Z, Snyder M, Dermitzakis ET, et al: Identification and analysis of functional elements in 1\% of the human genome by the ENCODE pilot project. Nature 447: 799-816, 2007.

14. Rosenfeld N, Aharonov R, Meiri E, Rosenwald S, Spector Y, Zepeniuk M, Benjamin H, Shabes N, Tabak S, Levy A, et al: MicroRNAs accurately identify cancer tissue origin. Nat Biotechnol 26: 462-469, 2008.

15. Rosenwald S, Gilad S, Benjamin S, Lebanony D, Dromi N, Faerman A, Benjamin H, Tamir R, Ezagouri M, Goren E, et al: Validation of a microRNA-based qRT-PCR test for accurate identification of tumor tissue origin. Mod Pathol 23: 814-823, 2010.

16. Jeong Y, Xie Y, Xiao G, Behrens C, Girard L, Wistuba II, Minna JD and Mangelsdorf DJ: Nuclear receptor expression defines a set of prognostic biomarkers for lung cancer. PLoS Med 7: e1000378, 2010.

17. Lu Y and Han J: Cancer classification using gene expression data. Information Systems 28: 243-268, 2003.

18. Gentleman RC, Carey VJ, Bates DM, Bolstad B, Dettling M, Dudoit S, Ellis B, Gautier L, Ge Y, Gentry J, et al: Bioconductor: Open software development for computational biology and bioinformatics. Genome Biol 5: R80, 2004.

19. Sherafatian M: Tree-based machine learning algorithms identified minimal set of miRNA biomarkers for breast cancer diagnosis and molecular subtyping. Gene 667: 111-118, 2018.

20. Colaprico A, Silva TC, Olsen C, Garofano L, Cava C, Garolini D, Sabedot TS, Malta TM, Pagnotta SM, Castiglioni I, et al: TCGAbiolinks: An R/Bioconductor package for integrative analysis of TCGA data. Nucleic Acids Res 44: e71, 2015.

21. Cancer Genome Atlas Research Network: Comprehensive molecular profiling of lung adenocarcinoma. Nature 511: 543-550, 2014.

22. Cancer Genome Atlas Research Network: Comprehensive genomic characterization of squamous cell lung cancers. Nature 489: 519-525, 2012.

23. Gentleman R, Carey V, Huber W and Hahne F: Genefilter: Methods for filtering genes from microarray experiments. $\mathrm{R}$ package version 1(0) $\mathrm{R}$ package version 1.42.0., 2011.

24. Kuhn M: Building predictive models in $\mathrm{R}$ using the Caret package. J Stat Software 28: 1-26, 2008.

25. Risso D: EDASeq: Exploratory data analysis and normalization for RNA-Seq, 2013.

26. Risso D, Ngai J, Speed TP and Dudoit S: Normalization of RNA-seq data using factor analysis of control genes or samples. Nat Biotechnol 32: 896-902, 2014.

27. Love MI, Huber W and Anders S: Moderated estimation of fold change and dispersion for RNA-seq data with DESeq2. Genome Biol 15: 550, 2014. 
28. Hao M, Wang Y and Bryant SH: An efficient algorithm coupled with synthetic minority over-sampling technique to classify imbalanced PubChem BioAssay data. Anal Chim Acta 806: 117-127, 2014.

29. Torgo L: Data mining with R: Learning with case studies, 2016: CRC press, Boca Raton, FL, USA.

30. Williams GJ: Rattle: A data mining GUI for R. R J 1: 45-55, 2009

31. Therneau TM, Atkinson B and Ripley MB: The rpart package. 2010.

32. Robin X, Turck N, Hainard A, Tiberti N, Lisacek F, Sanchez JC and Müller M: pROC: An open-source package for R and S+ to analyze and compare ROC curves. BMC Bioinformatics 12: 77, 2011.

33. Wang Y, Zhang X, Liu L, Li H, Yu J, Wang C and Ren X: Clinical implication of microrna for lung cancer. Cancer Biother Radiopharm 28: 261-267, 2013.

34. Feng B, Zhang K, Wang R and Chen L: Non-small-cell lung cancer and miRNAs: Novel biomarkers and promising tools for treatment. Clin Sci (Lond) 128: 619-634, 2015.

35. Sun Y, Bai Y, Zhang F, Wang Y, Guo Y and Guo L: miR-126 inhibits non-small cell lung cancer cells proliferation by targeting EGFL7. Biochem Biophys Res Commun 391: 1483-1489, 2010.

36. Zhong M, Ma X, Sun C and Chen L: MicroRNAs reduce tumor growth and contribute to enhance cytotoxicity induced by gefitinib in non-small cell lung cancer. Chem Biol Interact 184: 431-438, 2010.

37. Su Y, Guarnera MA, Fang H and Jiang F: Small non-coding RNA biomarkers in sputum for lung cancer diagnosis. Mol Cancer 15: $36,2016$.

38. Võsa U, Vooder T, Kolde R, Vilo J, Metspalu A and Annilo T: Meta-analysis of microRNA expression in lung cancer. Int J Cancer 132: 2884-2893, 2013.
39. Boeri M, Verri C, Conte D, Roz L, Modena P, Facchinetti F, Calabrò E, Croce CM, Pastorino U and Sozzi G: MicroRNA signatures in tissues and plasma predict development and prognosis of computed tomography detected lung cancer. Proc Natl Acad Sci USA 108: 3713-3718, 2011.

40. Lebanony D, Benjamin H, Gilad S, Ezagouri M, Dov A, Ashkenazi K, Gefen N, Izraeli S, Rechavi G, Pass H, et al: Diagnostic assay based on hsa-miR-205 expression distinguishes squamous from nonsquamous non-small-cell lung carcinoma. J Clin Oncol 27: 2030-2037, 2009.

41. Lu Y, Govindan R, Wang L, Liu PY, Goodgame B, Wen W, Sezhiyan A, Pfeifer J, Li YF, Hua X, et al: MicroRNA profiling and prediction of recurrence/relapse-free survival in stage I lung cancer. Carcinogenesis 33: 1046-1054, 2012.

42. Zhang JG, Wang JJ, Zhao F, Liu Q, Jiang K and Yang GH MicroRNA-21 (miR-21) represses tumor suppressor PTEN and promotes growth and invasion in non-small cell lung cancer (NSCLC). Clin Chim Acta 411: 846-852, 2010

43. Hamamoto J, Soejima K, Yoda S, Naoki K, Nakayama S, Satomi R, Terai H, Ikemura S, Sato T, Yasuda H, et al: Identification of microRNAs differentially expressed between lung squamous cell carcinoma and lung adenocarcinoma. Mol Med Rep 8: 456-462, 2013.

44. Gregory PA, Bert AG, Paterson EL, Barry SC, Tsykin A, Farshid G, Vadas MA, Khew-Goodall Y and Goodall GJ: The miR-200 family and miR-205 regulate epithelial to mesenchymal transition by targeting ZEB1 and SIP1. Nat Cell Biol 10: 593-601, 2008.

45. Duan B, Guo T, Sun H, Cai R, Rui Q and Xi Z: miR-205 as a biological marker in non-small cell lung cancer. Biomed Pharmacother 91: 823-830, 2017. 